\title{
Logical Tree and Complex Event Processing in Power Systems
}

\author{
Juntao Li \\ Peking University \\ Beijing, China \\ lijuntao@pku.edu.cn
}

\author{
Yibo Yang \\ Peking University \\ Beijing,China \\ iboing@163.com
}

\author{
Yu Huang \\ Peking University \\ Beijing,China \\ hy@pku.edu.cn
}

\author{
Yiming Lu \\ Department of power \\ distribution network China \\ Electric Power Research \\ Institute Beijing, China \\ luyiming@epri.sgcc.com.cn
}

\begin{abstract}
In virtue of the sharp increasing scale of distribution network and remarkable improvement of network complexity of power system, it is more challenging to monitor the actual running situation of the power network and to diagnose fault. To add insult to injury, man-made operation mistakes and inadequate situational awareness push the power system into a tight corner. This research presents a new approach, which could achieve efficient and sufficient situation awareness. Firstly, tree form structure was adopted in this model. Then the complex events processing engine is introduced. As a result, a comprehensive model is proposed in this paper, through taking data-based knowledge and model-based information into account. In the end, the software framework and results of simulation experiment is provided
\end{abstract}

Keywords—situation awareness; complex events processing; trees; power systems; data-based model

\section{INTRODUCTION}

Mounting number of network nodes at present have threatened the overall stability of power system, posing a conundrum for researchers to monitor various faults. There are already numerous incidents all over the world that are relative to power systems, one of which is the large-scale outages happened on august fourteen, 2003 in North America, as a result of which tens of millions have been affected and even paralyzed the urban public transit [1]. Another case of blackout occurred in September 2003 in Italy which had grabbed the news headlines for a period of time [2]. The most important element in these cases is devoid of real-time diagnoses and precise situational awareness in system of power networks. Accordingly, methodologies that are intimately bound up with situation awareness. Apart from situation awareness models, decision-making support methods offer effective tools for researchers and operators. With regard to decision-making support methods, decision tree algorithm is the most commonly used in practice, consisting of two main categories of trees: regression trees and classification trees [3]. The application of such a tree structure makes it possible to accomplish highly efficient and accurate anomaly detection and further rapid classification as well as ultimate predictions.

DOI reference number: 10.18293/SEKE2017-189

This work was supported by the Major State Research Development Program of China(2016QY04W0804)
In view of this, a new model of software architecture compatible with the existing power systems was proposed in this research. The main contents of this paper are as follows: the first part introduced the background information of situation awareness and its application in power system; the second part of the paper discussed related works, including implementation of regression trees and decision tress in power system, modelbased approaches of situation awareness, data-driven algorithms, generic frame-structure and practical tools; in the third section, a reasonable and complete definition of situation awareness has been given; in part four, the process of situation in power system was been illustrated; in part five, specific algorithm of situation awareness was proposed; the six part of this paper indicate the results of simulation experiment; the seven part of this study is the conclusion that sums up this work and mentions a few problems to be resolved.

\section{RELATED WORK}

The theories and problems as well as application of SA in power networks have been researched, consisting of the sources of inadequate SA, an information system that is applicable to complex system, and a general architecture of software. In study [4], the standards and tools of SA in power networks have also been given, as a result of which perception levels of system operators were enhanced obviously. From another perspective, a novel approach, a typically data-driven theory, has been introduced to cognize the status of power networks, which is mainly based on the random matrix theory [5]. Another key technology achieved in electrical networks is decision-making tree due to the fact that it possesses a unique characteristic compared with other machine learning algorithms $[6,7,8,9]$. Meanwhile, the performance estimation of CEP in real-time network monitoring turned out to be very attractive, since it has simultaneously real-time access to data, fast speed query, and effective data reduction [10]. In this research, hence, a novel method that is a combination of CEP technologies and effective trees inference structure showed excellent capability of situation awareness in power systems. Meanwhile, through taking into account information obtained by data-driven approach, a variety of hierarchies of control information is quickly pushed to corresponding users in power systems. 
Besides, another definition of situation awareness in power gird was given in this paper and also the software based on framestructure illustrated later was accomplished.

\section{DeFinAtion OF SituAtion AWARENESS}

Situational awareness reasoning model for distribution network was built by using the combination of Analytic hierarchy process (AHP) and B-tree-like structure, and users can also evolve this model. Through taking different rules sets, the reasoning process of model can be achieved from down to up and vice versa, to implement disaster situational awareness and trace corresponding resources in the converse process. Moreover, when it comes to the process of modeling, each element in each of the AHP layers is defined as a single node. By connecting all the nodes, the B-tree for situational awareness was built. Fig. 1 showed the object of model elements.

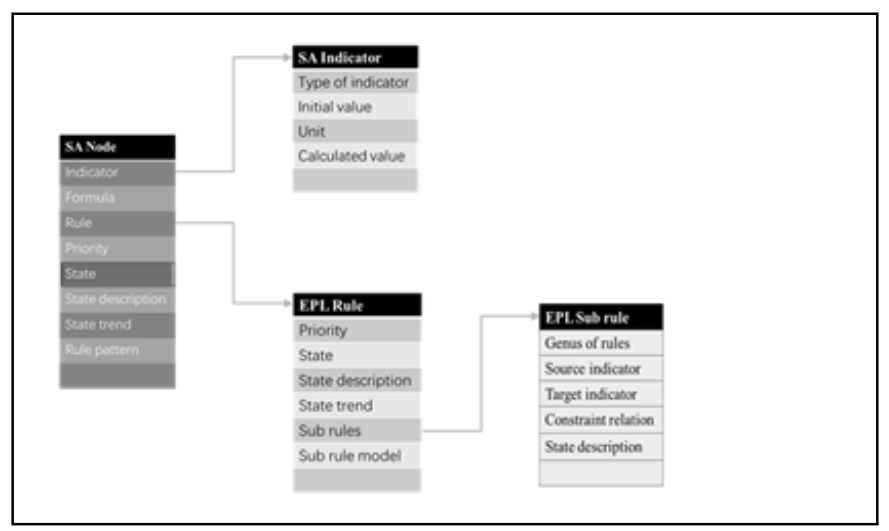

Figure 1. The object of model elements includes nodes, indicators, rules and sub-rules

In addition, relative data normalization of situational awareness is as follows. First, basic elements and constraints of the construction of the frame are defined, which each basic element represent element model (node). Eventually, tree of perception model was constructed through using rules to connect individual nodes. Fig. 1 showed structural design of each node, and structure of rules and indicators.

Constraints of nodes are as follows: each node contains one or more rules; each rule comprises one or more sub-rules; there exists only one relation of 'and', 'or' between rules in the same layer, so do sub-rules; state values of rules and sub-rules consist of two types: true and false; through applying 'and' operation and 'or' operation in the same layer, the state value of sub-rules and rules could be aggregated into the state values of upper layer; reasoning and judgment of sub-rules is based on the statues values by comparing values of source indicators and target indicators; the function of and-or-invert could be reasonably decomposed into combination of 'and' and 'or' operation.

The essence of situation awareness model is ontology model and knowledge, which makes it possible that users could create the classification of situational awareness model with the model library. Users can manage their own ontology model and knowledge.

Situation awareness model contain the following attributes: meta-model knowledge, ontology knowledge of rules, ontology knowledge of indicators, other attributes description. Ontology knowledge of rules can also include knowledge of sub-rules, formula, and indicators. Ontology knowledge of formulas can also contain sub-formulas and indicators. The system further abstracted rules into atomic events and composite events. While formulas are used as the expression of events detection, indicators are defined as monitoring parameters of events detection.

\section{PROCESS OF SitUATION AWARENESS}

Dynamic hierarchical model in early warning is based upon analytic hierarchy process and trees structure, which build the relationship between key factors and nodes of the tree. As a result, hierarchical early warning model of power grid security can be established, which can be used for dynamic hierarchical warning of power system risk. Flow diagram of situation awareness is indicated in Fig. 2.

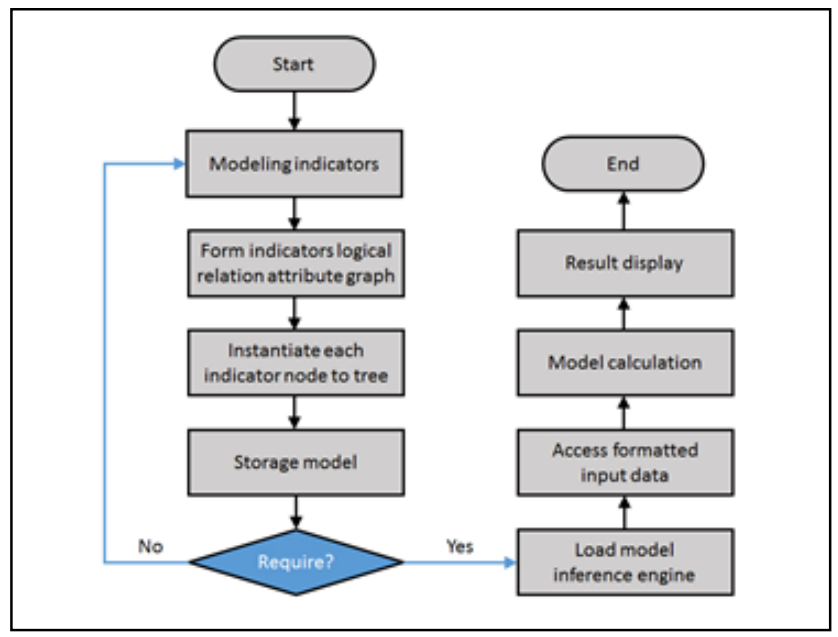

Figure 2. The flow diagram of situation awareness

This model consists of the following steps:

1) Establish the indicators system of power networks security risk early warning: According to the collected data in the monitoring system of power networks, the indicators preliminarily selected such as natural disasters and man-made mistakes.

2) Construct the graph of tree structure with layered nodes and child nodes that is corresponding to stratification of regions: Each leaf node of the tree represents a county. Their parent nodes represent corresponding cities which are larger regions than counties, while the region of a province is expressed as the root node.

3) Set the indicators and formulas as well as hierarchical model of power networks security risk early warning involved in each node for each single layer of the tree: The setup method of hierarchical model includes the following steps: The first stage is to define variables, and variable valuations are obtained from further calculation by using those relative formulas. The hierarchical model can be optimized on account of variable valuations, which the layers of hierarchical model are consistent with regional layers. This model is established and stored. The model that has already been stored also supports modification operations. 
4) Each node of hierarchical model is marked by different colors:In practical application, the implementation of the operations are as follows: The object of previously created early-warning model is initialized with tree structure, and the object of indictors in the warning model is also loaded in memory; Certain types of data are obtained from the detection system of power gird as input data; Matching nodes of input data are found according to different indicators; input data can be computed use formulas within coincide node; various values are labeled as different colors by means of rule-based judgement of each node; different colors represent different levels of risk; red color indicates that the node is at risk, while green color indicates devoid of risk; when a specific node of a layer turns red, homologous risks are warned.

\section{SPECIFIC ALGORITHMS}

\section{A. Complex Event Processing Based Situational Awareness}

The situation in the short term and ultra-short term can be predicted on account of the result of data understandability. Predicting consequences of relatively low level is primarily focused on deterministic prediction in the case of a single fault, while higher level yield the probabilistic load forecasting results of all possible circumstance. Deterministic prediction presents exact single-point prediction, while probabilistic prediction provides confidence level and probability of predictable variables.

In addition, the situation awareness is implemented in the rolling way which means predicting outcomes in the past would be the present situation as time goes by. Therefore, future predicting consequences should be amended by current results of data cognition and situation awareness.

\section{B. State Machine Processing}

Detection and monitoring of composite events are implemented based on the combination of rules, indicators and initial values. Workflow-based data is propagated to real-time indicator data of each threshold node. Current situation is acquired from both model and data. The approach of complex event processing is used to analyze the variation trend and relationship of data for the reason of achieving situational predication. Through taking the current and future state of the model into account, users can set up rules and model to realize intelligent early-warning.

\section{Establishment of Inference Rules}

Inference rules can be abstracted as events in the system, as a result of which atomic events and composite events represent rules and rule set respectively. The implementation of rules is as follows: ontology knowledge of rules is established; Meta knowledge and essential attributes are maintained; indicators are added to rules; indicators names and initial values as well as description information are maintained, which the indicators are available from indicator base or user-defined indicators; rules bind to sub-rules, which all these rules are selected from existing library. Judgment outcomes and status value (true/false) of rules and sub-rules are the main reference of state changes, which status values are obtained from the 'and/or' judgment of rules based on the node models. According to state values of rules or nodes, the node information of next level could be acquired so that situation reasoning and awareness could be accomplished, as illustrated in Fig. 3.

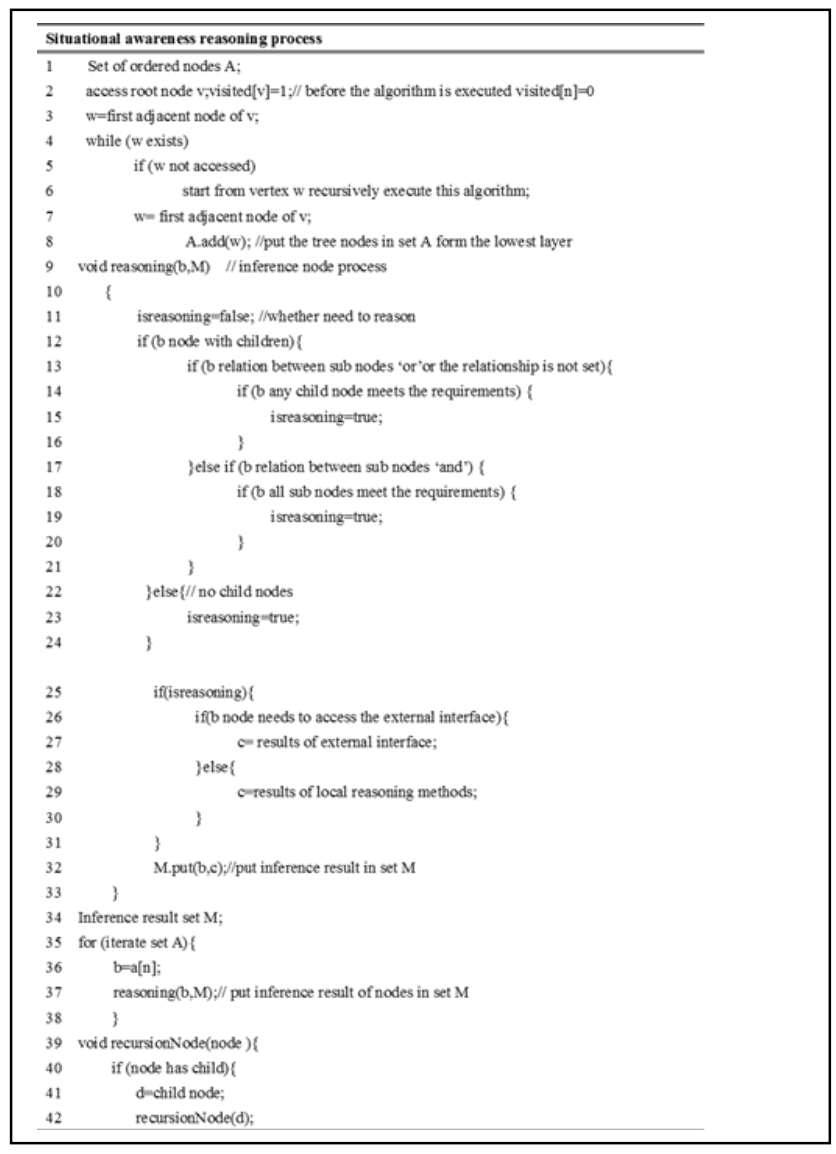

Figure 3. The specific algorithm of situation awareness

Knowledge can be adversely traced back to its sources on the basis of the reasoning results of the model. And relevant knowledge of rules and indicators as well as meta-knowledge in the process of inference could be adversely traversed and can be presented to the user. Through considering the correspondence between complex events and specific rules, and saved incidence relation between knowledge of rules and ontology knowledge, all of the knowledge can be traced back. The algorithm of adverse tracing is given in Fig. 4.

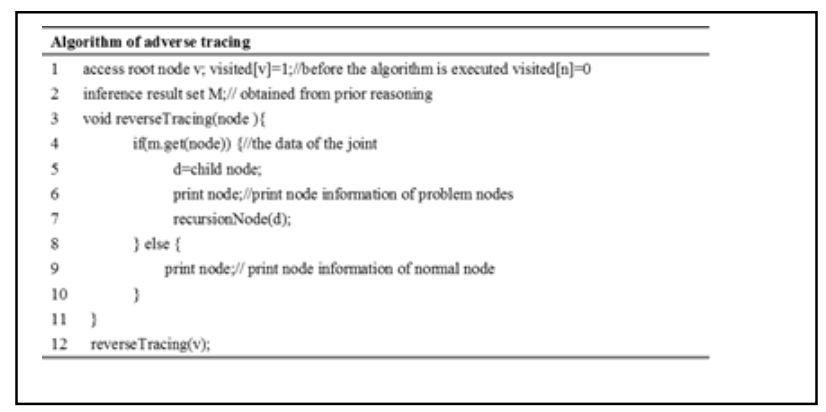

Figure 4. Algorithm of adverse tracing

\section{SIMULATION EXOERIMENT}

Data for reasoning includes weather (temperature, wind velocity), power lines (length, type): Rule of node wire length is that the length of wires is more than 10 kilometers; rules of 
node temperature is that the temperature is below three degrees; nodal wind speed is greater than $7 \mathrm{~m} / \mathrm{s}$; the type of power wires is overhead line; overhead lines icing will occur when the length of overhead lines is greater than 12 kilometers and wind speed is greater than $10 \mathrm{~m} / \mathrm{s}$.

Data rules: Incoming weather data:

$\{$ "data": \{ "weather":[\{ "ID":"weatherID1","temp":1,"winds peed":6,"eleLineID":"eleLinedID1"\}] \}, "finish":"false" \}

Incoming wires data:

$\{$ "data": \{ "eleLine":[\{"ID":"eleLinedID1","len":150,"overh ead":1\}]\},"finish":"true" $\}$

'finish' represents whether the data is introduced completely. If completed, reasoning process is started. The reasoning results are shown in Fig. 5.

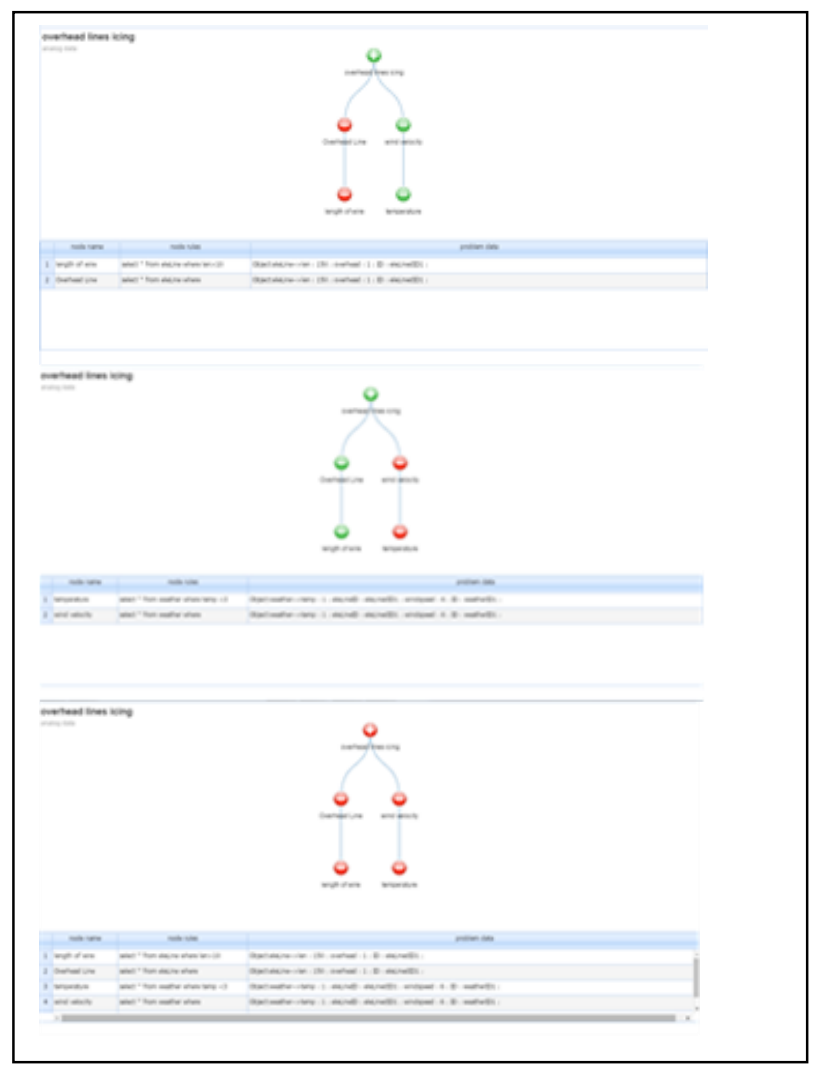

Figure 5. From top to bottom, it displays the problems of overhead lines, temperature, and both wire and temperature, respectively.

\section{CONCLUSION}

In this study, a universal framework that is compatible with complex and large-scale power networks has been proposed. This framework not only adopts model-based approaches of situation awareness, but also data-driven models of SA, as a result of which, adequate cognition of electric power networks status is obtained. According to theoretical analysis, accidents caused by man-made operation mistakes and insufficient SA is reduced significantly by this framework. And it consists of cloud storage, generation of information system, situation awareness algorithms. Another formal definition of situation awareness in power systems is descripted in this paper. When it comes to the specific algorithms of situation awareness in this study, the Esper engine is used for processing complex events stream, which is only part of the situation awareness algorithm, instead of using decision trees or single method of CEP. Using the information of practical systems gathered from Esper engine as a single node, a logic tree is trained for representing information and achieving further situational awareness.

Tree structures offer advantages of fast inquiry, swift feedback, effective decision-making, and the ability to rapidly response adventitious developments. Insomuch as multigranularity SA information can be extracted from the tree structure, it would be wiser to push corresponding fault warning information and decision-making information into system operators with different priorities. Construction method of storage also is elaborated in this study owing to the fact that the function of real-time access and inquiry of data system has important engineering significance. In the end, the technology of visualization is studied. The software development of this framework has already been completed and simple simulative experiments are carried out.

However, there are a few problems to be solved in this research. For one thing, in the absence of real datasets, it is impossible to achieve further cost evaluation and performance tests. In future work, key performance indicators of the system such as response time, reliability, and accuracy would be experimented and confirmed by using real data. For another, distributed deployment of this model would be accomplished in order to bring a better experience the users.

\section{ACKNOWLEDGEMENT}

$\mathrm{Yu}$ Huang is the corresponding author of this paper.

\section{REFERENCES}

[1] Force U C P S O T, Abraham S, Dhaliwal H, et al. Final Report on the August 14, 2003 Blackout in the United States and Canada: Causes and Recommendations, Natural Resources Canada, Ottawa[J]. 2004.

[2] Interim Report of the Investigation Committee on the 28 September 2003 Blackout in Italy, UCTE, 2003.

[3] Diao R, Vittal V, Logic N. Design of a Real-Time Security Assessment Tool for Situational Awareness Enhancement in Modern Power Systems[J]. IEEE Transactions on Power Systems, 2010, 25(2):957-965.

[4] Panteli M, Kirschen D S. Situation awareness in power systems: Theory, challenges and applications[J]. Electric Power Systems Research, 2015, 122:140-151.

[5] He X, Qiu R C, Ai Q, et al. Linear Eigenvalue Statistics: An Indicator Ensemble Design for Situation Awareness of Power Systems[J]. Statistics, 2015.

[6] Wehenkel L, Cutsem T V, Ribbens-Pavella M. An artificial intelligence framework for online transient stability assessment of power systems[J]. IEEE Transactions on Power Systems, 1989, 4(2):789-800.

[7] Sun K, Likhate S, Vittal V, et al. An Online Dynamic Security Assessment Scheme Using Phasor Measurements and Decision Trees[J]. IEEE Transactions on Power Systems, 2007, 22(4):1935-1943.

[8] Diao R, Vittal V, Logic N. Design of a Real-Time Security Assessment Tool for Situational Awareness Enhancement in Modern Power Systems[J]. IEEE Transactions on Power Systems, 2010, 25(2):957-965.

[9] Senroy N, Heydt G T, Vittal V. Decision Tree Assisted Controlled Islanding[J]. Power Systems IEEE Transactions on, 2006, 21(4):17901797.

[10] Balis B, Kowalewski B, Bubak M. Real-time Grid monitoring based on complex event processing [J]. Future Generation Computer Systems, 2011, 27(8):1103-1112. 